\title{
Charged Higgs boson searches at the LHC
}

Dhiman Chakraborty $\begin{array}{ll}\text { Northern Illinois } \\ \text { NII }\end{array}$

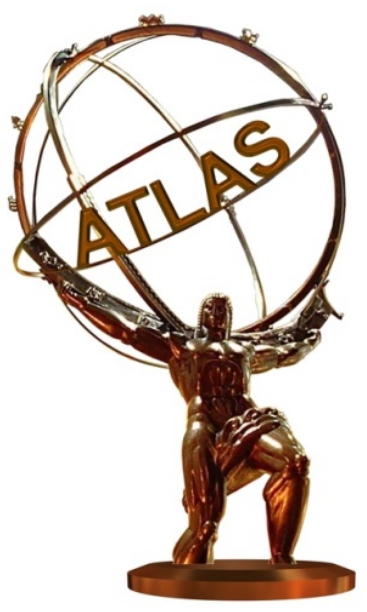

\author{
On behalf of the \\ ATLAS and CMS experiments
}

The 13th International Workshop on Tau Lepton Physics Aachen, Germany, 15-19 September, 2014 


\section{Outline}

- Motivation

- Phenomenology

- Basic postulates and parameters

- Couplings and decay characteristics

- Production at the LHC

- Experimental search

- Strategies for various production and decay channels:

signal characteristics, background, challenges

- Results

- Summary and outlook 


\section{Motivation}

- Discovery of $\underline{a}$ Higgs boson prompts further investigations into the scalar sector - it may well be richer in structure than the minimum required by the SM.

- Charged Higgs bosons appear in many extensions of the SM.

- A "Type 2" two-Higgs doublet model (2HDM) is an essential feature of the MSSM.

- It is strategically straightforward to look for deviations from SMpredicted fermion-mass-independence in charged-current couplings, especially in top quark production and decay. 


\section{The two-Higgs-doublet model (2HDM)}

- Two complex doublet scalar (Higgs) fields with opposite hypercharge.

- Of the 8 degrees of freedom, 3 are expended in giving mass to $W^{ \pm}, Z$.

- The remaining 5 are manifested as physical particles:

- two neutral scalars $\left(\mathrm{h}^{0}, \mathrm{H}^{0}\right)$,

- one neutral pseudoscalar (A),

- one charge-conjugate scalar pair $\left(\mathrm{H}^{+}, \mathrm{H}^{-}\right)$.
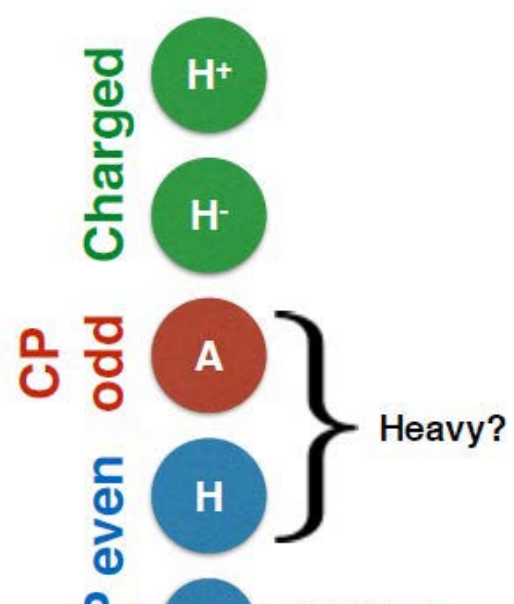

○ h sM-like?

- In "Type 2" 2HDM, one complex doublet couples with up-type quarks (and neutrinos), the other with down-type quarks and charged leptons.

- The two parameters of the model are commonly chosen as the ratio of the two vacuum expectation values and the mass of either $\mathrm{A}$ or $\mathrm{H}^{+}$. 


\section{$\mathrm{H}^{+}$coupling with fermions in Type 2 2HDM}

- $\tan \beta=\frac{v_{2}}{v_{1}}$

$m_{W}^{2}=\left(v_{1}^{2}+v_{2}^{2}\right)$

- $\mathcal{L}\left(H^{+} U D\right)=$

$\frac{g}{2 \sqrt{2} m_{W}}\left(H^{+} U K\left(m_{U} \cot \beta\left(1-\gamma_{5}\right)+m_{D} \tan \beta\left(1+\gamma_{5}\right) D\right)+\right.$ h.c.

- Current $\mathrm{H}^{+}$searches at LHC are focused on production and decay via interactions with SM fermions.

- $H^{+} t b$ coupling is the strongest of all irrespective of $\left(m_{H^{+}}, \tan \beta\right) \rightarrow$ Production most likely in decay of top quarks if $m_{H^{+}}<m_{t}$, otherwise in association with the top quark.

- Decay driven by phase space $\left(m_{H^{+}}\right)$and $\tan \beta$. 


\section{$\mathrm{H}^{+}$decay branching fractions}

Partial decay widths calculated with FeynHiggs and HDecay (using MSSM input parameters provided by FeynHiggs)

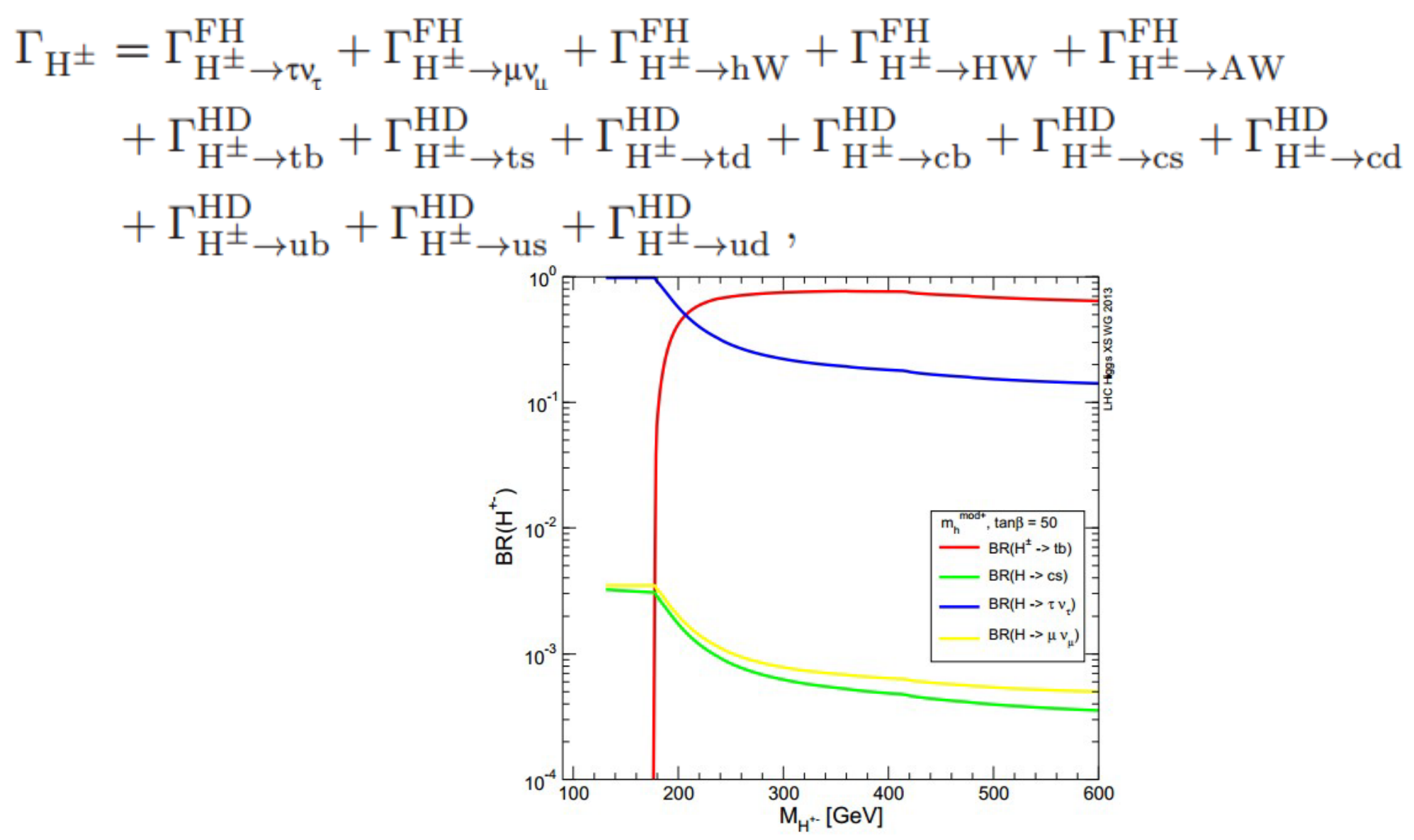

https://twiki.cern.ch/twiki/bin/view/LHCPhysics/BRs\#YR3_numbers 


\section{Previous results}

No evidence of $\mathrm{H}^{+}$production found so far. $95 \% \mathrm{CL}$ limits:

- LEP combined:

- $m_{H^{+}}>78.6 \mathrm{GeV}$ irrespective of model.

- $m_{H^{+}}>90 \mathrm{GeV}$ assuming Type $2 \mathrm{HDM}, B\left(H^{+} \rightarrow \tau^{+} v_{\tau}\right)=1$.

- Tevatron (D0, CDF):

- $B\left(t \rightarrow H^{+} b\right)<[0.15-0.20]$ depending on $m_{H^{+}}, \tan \beta$, Type 2 2HDM assumed. 


\section{The LHC}

\section{Large Hadron Collider}

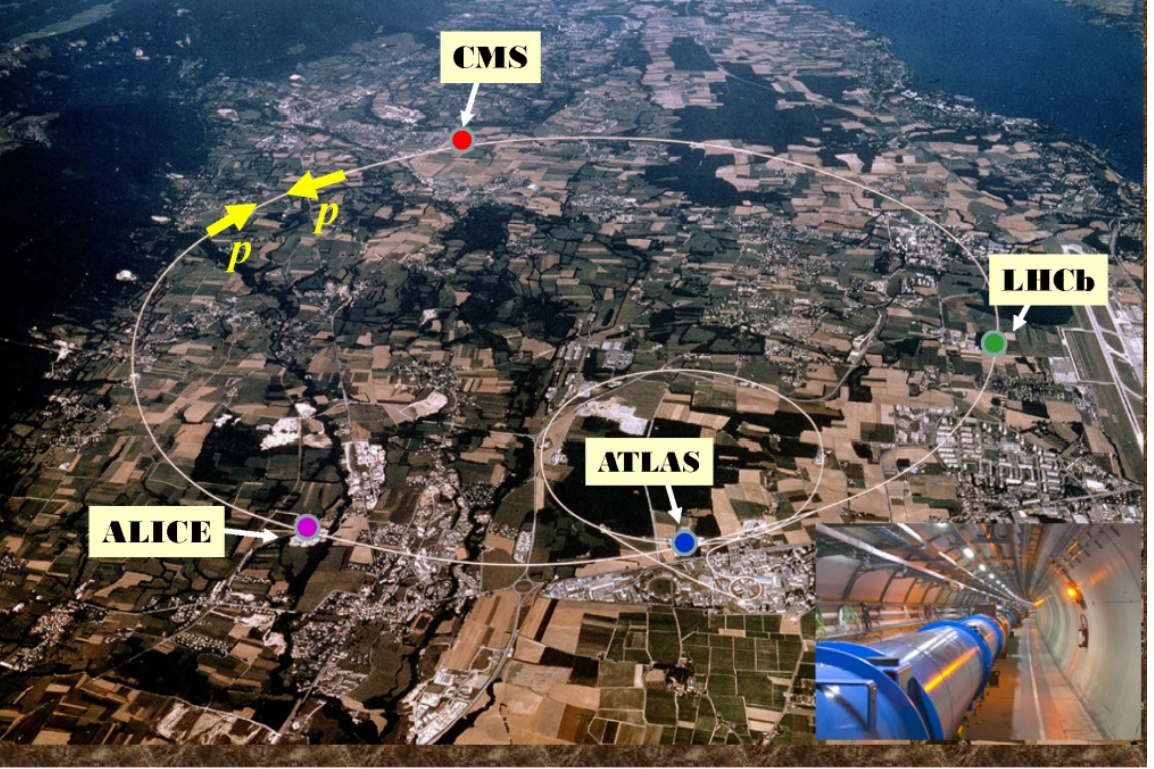

- Peak lumi $\sim 7.7 \times 10^{33} \mathrm{~cm}^{-2} \mathrm{~s}^{-1}$

- On avg, 21 interactions/crossing in $2012 \rightarrow$ large pile-up pp collider designed for $\sqrt{S}=14 \mathrm{TeV}$. Delivered so far:

$$
\begin{aligned}
& -\sim 5.4 \mathrm{fb}^{-1} \text { at } \sqrt{s}=7 \mathrm{TeV} \text { (2011) } \\
& \text { - } \sim 23 \mathrm{fb}^{-1} \text { at } \sqrt{s}=8 \mathrm{TeV} \text { (2012) }
\end{aligned}
$$

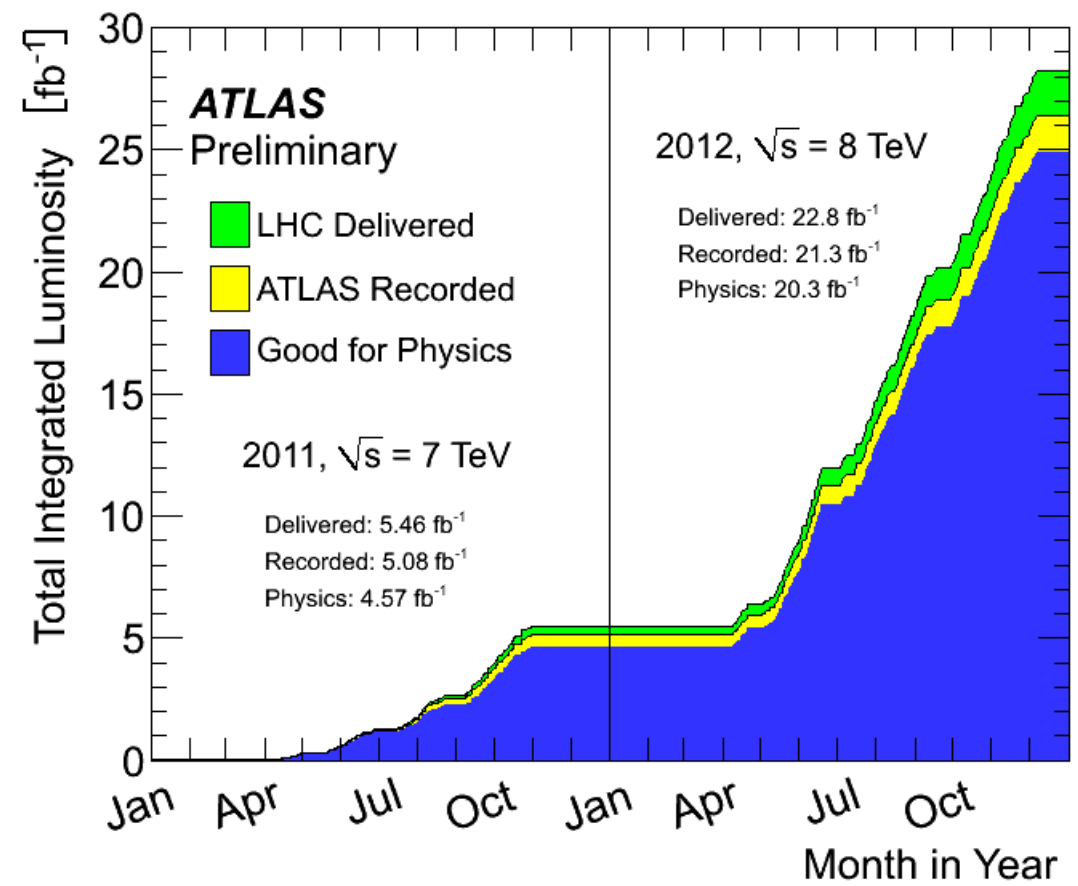




\section{$\mathrm{H}^{+}$production at the LHC: processes}

Relevant coupling: $g\left(t b H^{+}\right) \sim\left(m_{t} \cot \beta+m_{b} \tan \beta\right)$

If $\boldsymbol{m}_{H^{+}}<\boldsymbol{m}_{\boldsymbol{t}}$

Primary contribution from decays of top quarks

- Production cross section:

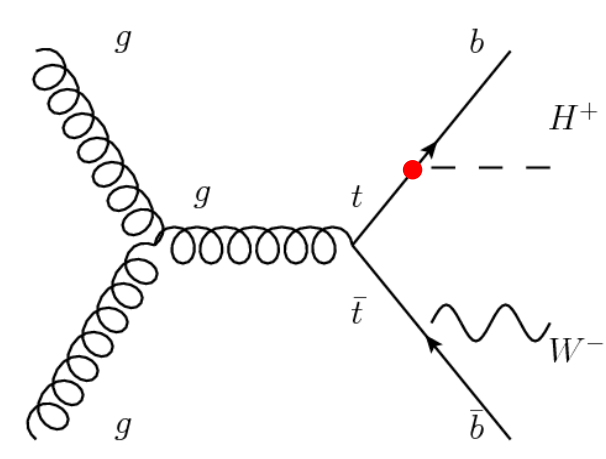

$\sigma(p p \rightarrow t \bar{t}) \times B\left(t \rightarrow H^{+} b\right) \quad$ [dominant at LHC]

The first factor is calculated and measured with

precision better than $10 \%$. There are several options to measure the latter.

\section{If $\boldsymbol{m}_{\boldsymbol{H}^{+}}>\boldsymbol{m}_{\boldsymbol{t}}$}

Production is expected to occur primarily in association with single top quarks - replace $W^{+}$
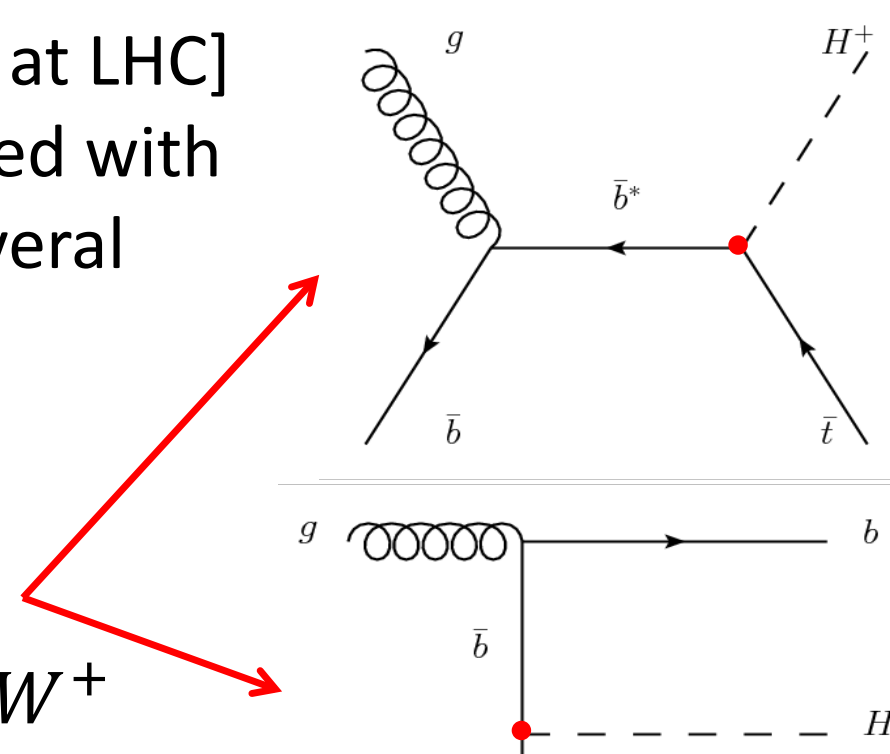
with $\mathrm{H}^{+}$in SM single top production.

- Production cross section is much smaller.

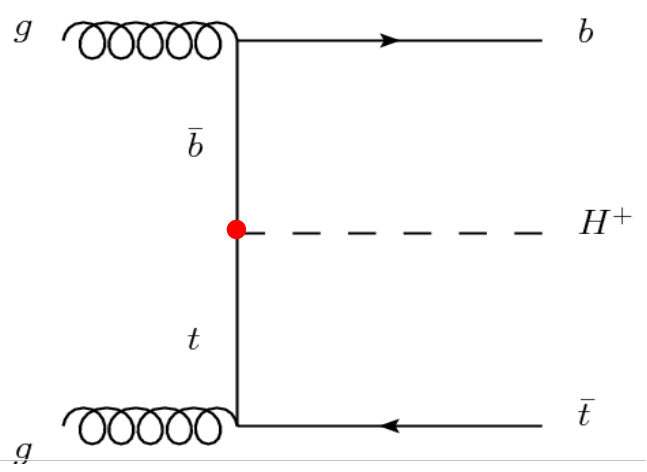




\section{$\mathrm{H}^{+}$production at the LHC: cross section, $m_{H^{+}}<m_{t}$}

Partial decay widths calculated with FeynHiggs and HDecay (using MSSM input parameters provided by FeynHiggs)
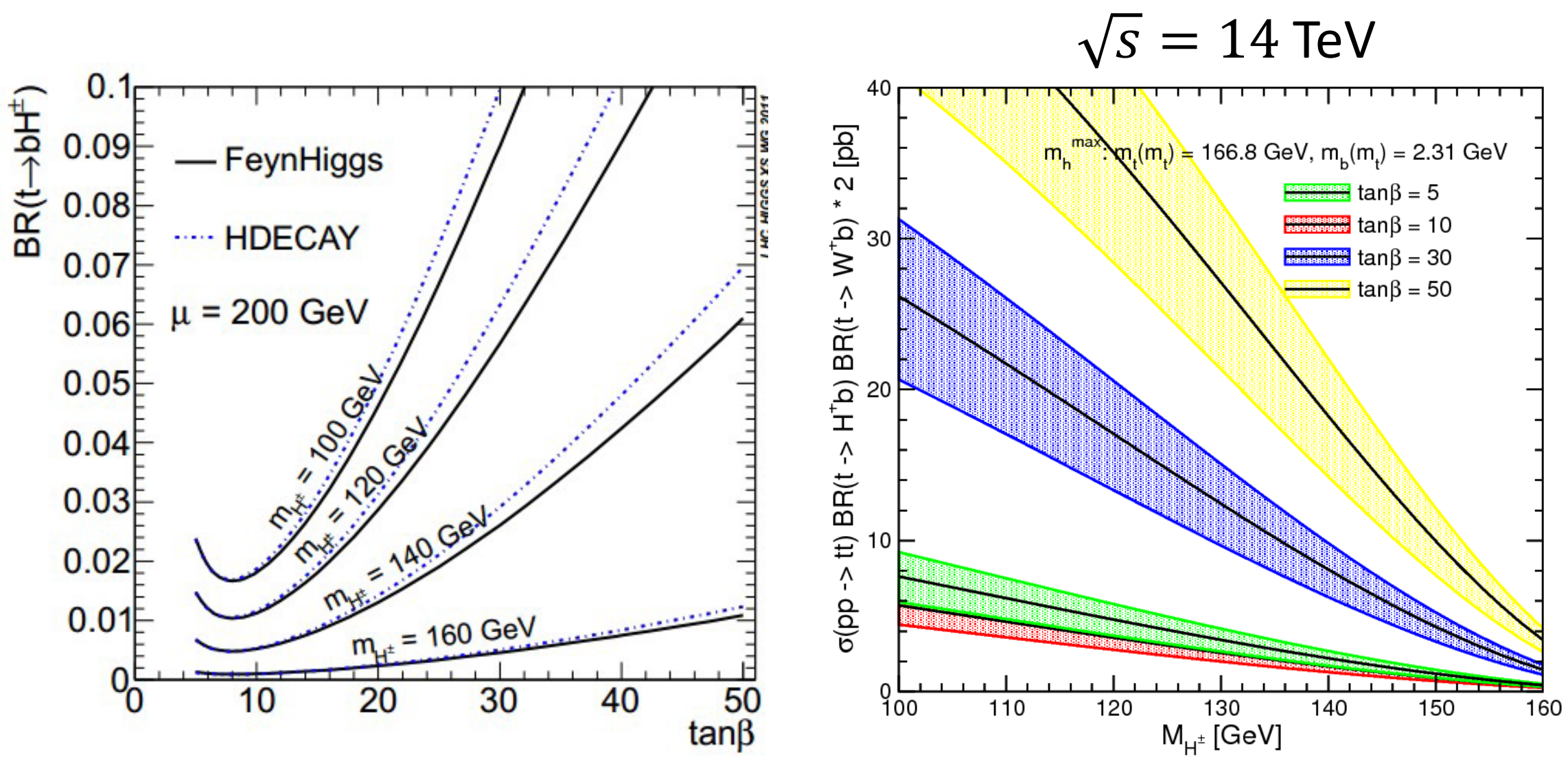

https://twiki.cern.ch/twiki/pub/LHCPhysics/MSSMCharged/mhmax-tb.tar.gz 


\section{$\mathrm{H}^{+}$production at the LHC: cross section, $m_{H^{+}}>m_{t}$}

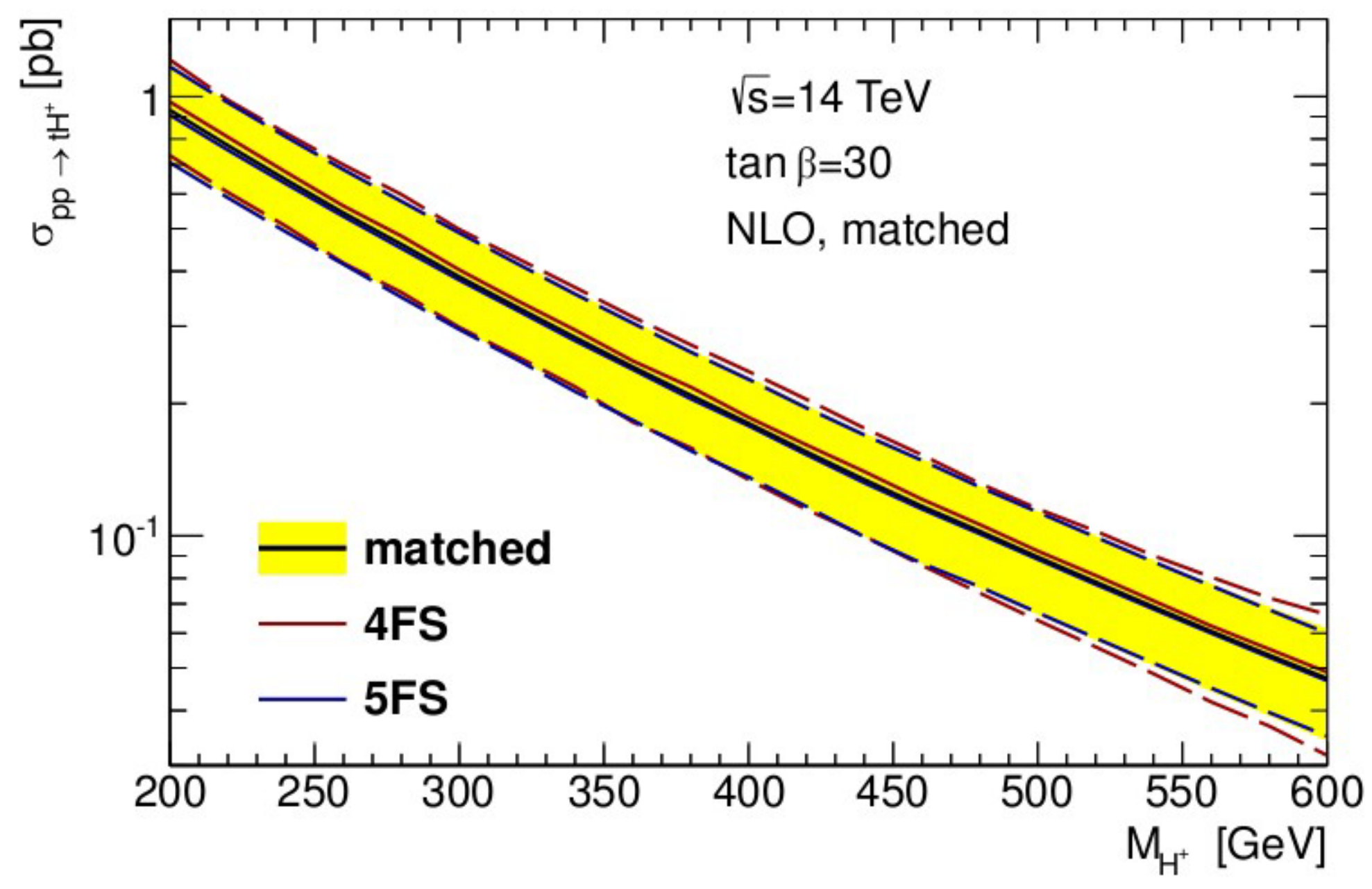

https://twiki.cern.ch/twiki/bin/view/LHCPhysics/MSSMCharged 


\section{$\mathrm{H}^{+}$search strategies at the $\mathrm{LHC}$}

For $\boldsymbol{m}_{\boldsymbol{H}^{+}}<\boldsymbol{m}_{\boldsymbol{t}}$

- Look for $t \rightarrow H^{+} b$ followed by

- $H^{+} \rightarrow \tau^{+} v_{\tau}$ for $\tan \beta>\sim 1$

- $H^{+} \rightarrow c \bar{s}$ for $\tan \beta<\sim 1$

- $B\left(t \rightarrow H^{+} b\right) \approx 0$ at $\tan \beta \approx \sqrt{\frac{m_{t}}{m_{b}}} \approx 7:$ sensitivity is at minimum

For $\boldsymbol{m}_{H^{+}}>\boldsymbol{m}_{\boldsymbol{t}}$

- $H^{+} \rightarrow t b \rightarrow W^{+} b \bar{b}$ dominates, but $H^{+} \rightarrow \tau^{+} v_{\tau}$ is cleaner with a branching fraction of $\sim 0.2$ if $\tan \beta>\sim 3$.

$H^{+} \rightarrow W^{+} b \bar{b}$ is significant even for $\tan \beta<\sim 1$ if $m_{t}-m_{H^{+}}$is small.

However, it is very difficult to extact from the SM decay of $t \bar{t}$. 


\section{Search strategies : $H^{+} \rightarrow \tau^{+} \boldsymbol{\nu}_{\boldsymbol{\tau}}$}

Look for a high- $p_{T} \tau$ decaying into hadrons

- $B\left(\tau_{h}\right) \approx 0.65$ with $\sim 0.5$ to "1-prong", $\sim 0.15$ to "3-prong".

- Collimated energy deposits with low track-multiplicity, often with identifiable EM contribution from $\pi^{0}$ s in $\tau$ decay.

Challenges:

- A good fraction of the $\tau$ momentum is carried away by the $v_{\tau}$ in decay. Only the remaining part is directly visible $\left(\tau_{v i s}\right)$.

- Multiple $v^{\prime}$ s in the event weakens the usefulness of $E_{T}^{\text {miss }}$.

- Large QCD background (quark- and gluon-initiated jets being misidentified as $\tau$ 's ). Hurts efficiency, especially at the trigger level, unless the event also features a high- $p_{T} e$ or $\mu$. 


\section{Search strategies : $\boldsymbol{H}^{+} \rightarrow \boldsymbol{c} \overline{\boldsymbol{S}}$}

Look for $t \bar{t}$ events with one top decaying into 3 jets: $t \rightarrow H^{+} b \rightarrow c \bar{s} b$

- $m_{j_{1} j_{2}}=m_{H^{+}}$

- $m_{j_{1} j_{2} j_{3}}=m_{t}$, possibly with $j_{3}=b$.

Challenges:

- In order to have any hope against the enormous QCD background, especially at the trigger level, the other top must decay into a high- $p_{T} e$ or $\mu$.

- Loss of discrimination if $m_{H^{+}}$is close to $m_{W^{+}}$.

- Competition from $H^{+} \rightarrow W^{+} b \bar{b}$ as $m_{H^{+}}$approaches $m_{t}$.

- Serious combinatorics from ISR, FSR, pile-up jets. 


\section{Other search strategies}

- Look for tell-tale violation of lepton universality in charged current interactions among $t \bar{t}$ event candidates,

- Look for $H^{0} \rightarrow H^{ \pm} W^{\mp}$ (Higgs "cascade" decays),

- Look for $H^{+} \rightarrow t \bar{b}$,

- Other all-bosonic vertices,

- Vertices involving BSM particles.

\section{(Results are available from the first three of these)}




\section{ATLAS and CMS}

\section{Two general-purpose detectors for the LHC}

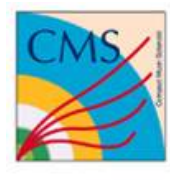

Detector characteristics

$\begin{array}{ll}\text { Width: } & 22 \mathrm{~m} \\ \text { Diameter: } & 15 \mathrm{~m}\end{array}$

Diameter:
Weight: $15 \mathrm{~m}$
14500t
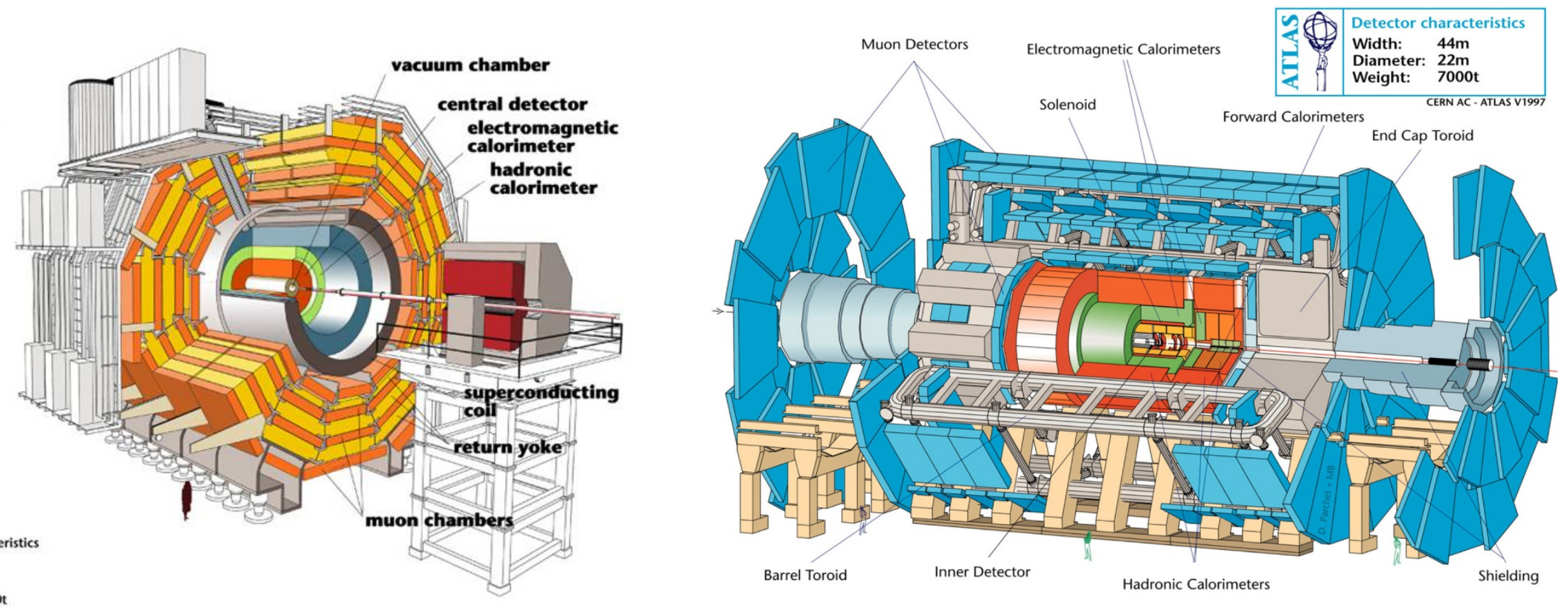

- Optimized to identify and measure electrons, photons, muons, taus, jets $(q / g)$, missing $p_{\mathrm{T}}$ with excellent resolution \& hermeticity.

- For offline analysis, multi-level triggering system select $\sim 200$ out of $\sim 40$ million bunch crossings every second. 


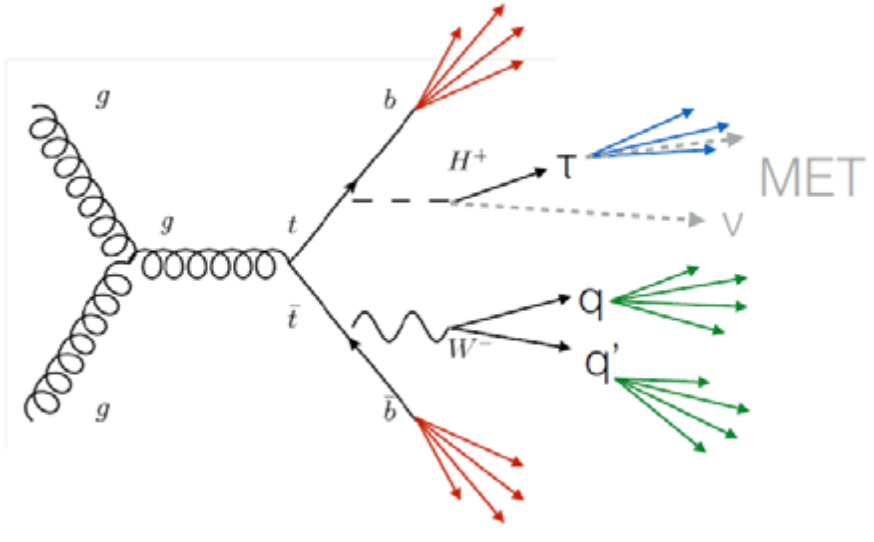

- Use $\tau+E_{T}{ }^{\text {miss }}$ trigger.

- Look for excess in $m_{T}\left(\tau, E_{T}^{\text {miss }}\right)$ distribution.

$$
m_{\mathrm{T}}=\sqrt{2 p_{\mathrm{T}}^{\tau} E_{\mathrm{T}}^{\mathrm{miss}}\left(1-\cos \Delta \phi_{\tau_{\text {had-vis }}, \text { miss }}\right)}
$$

- Dominant background: SM $t \bar{t}$, QCD multi-jet.
- For $m_{H^{+}}<m_{t}$ search, assume $B\left(H^{+} \rightarrow \tau^{+} v_{\tau}\right)=1$ to derive limits on $B\left(t \rightarrow H^{+} b\right)$.

- For $m_{H^{+}}>m_{t}$, use similar event selection, but allow other decay modes to derive limits on $\sigma\left(p p \rightarrow H^{ \pm}\right) \times B\left(H^{+} \rightarrow \tau^{+} v_{\tau}\right)$

\section{ATLAS-CONF-2014-050}




\section{$H^{+} \rightarrow \tau^{+} v_{\tau} ; m_{H^{+}}<m_{t}($ ATLAS)}
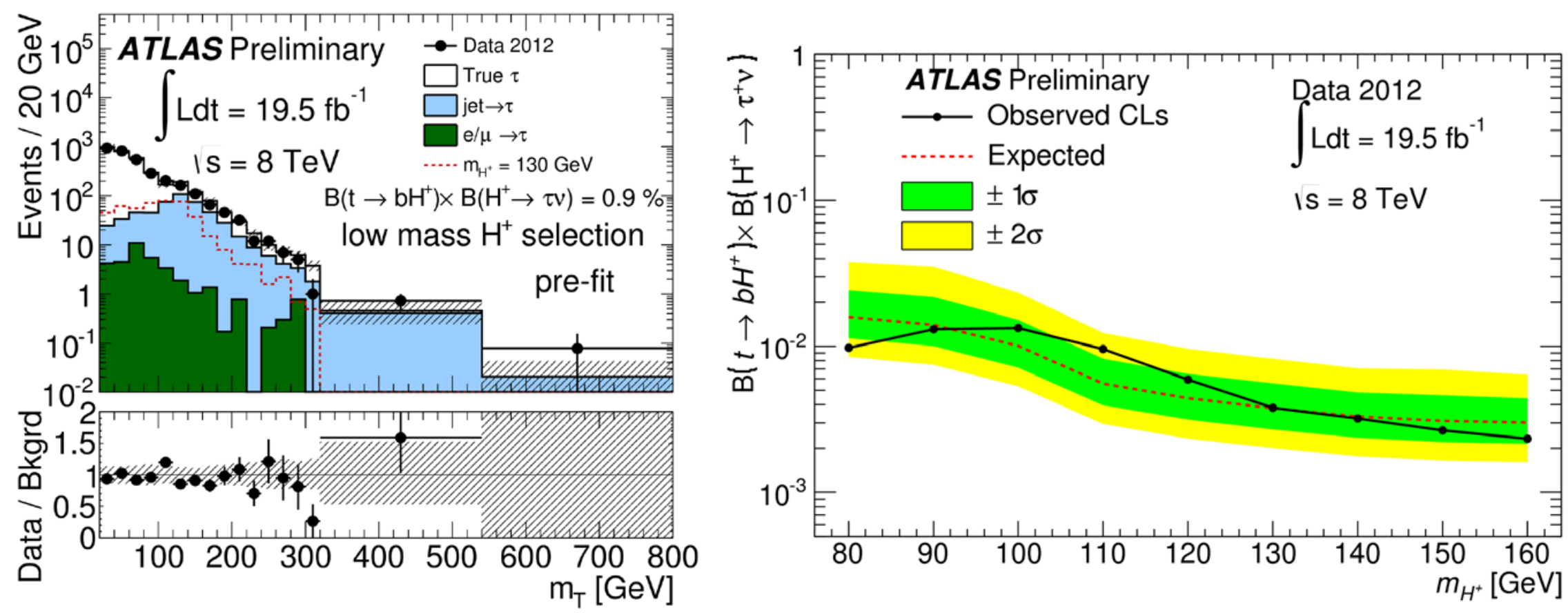

$B\left(t \rightarrow H^{+} b\right)<0.0023-0.013$ at $95 \% \mathrm{CL}$ for $80 \mathrm{GeV}<m_{H^{+}}<160 \mathrm{GeV}$

ATLAS-CONF-2014-050 


\section{$H^{+} \rightarrow \tau^{+} v_{\tau} ; m_{H^{+}}>m_{t}($ ATLAS)}
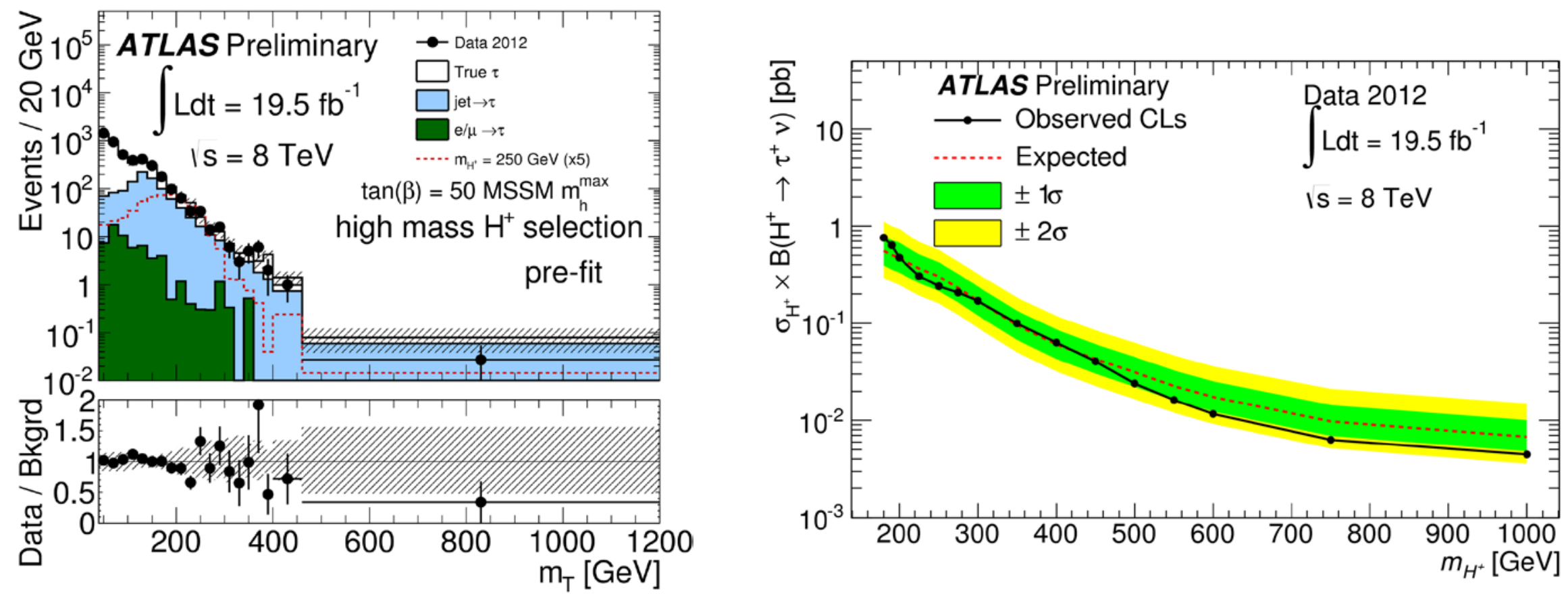

$$
\begin{gathered}
\sigma\left(p p \rightarrow H^{ \pm}\right) \times B\left(H^{+} \rightarrow \tau^{+} v_{\tau}\right)<0.004-0.76 \mathrm{pb} \text { at } 95 \% \mathrm{CL} \\
\text { for } 180 \mathrm{GeV}<m_{H^{+}}<1000 \mathrm{GeV}
\end{gathered}
$$

ATLAS-CONF-2014-050 


\section{$H^{+} \rightarrow \tau^{+} v_{\tau}$ or $t \bar{b} ; m_{H^{+}}>m_{t}(\mathrm{CMS})$}

- Combined search in the $\tau_{h} l$ and $l_{1} l_{2}$ final state $l_{(1,2)}=e, \mu$.

- Trigger on the isolated lepton $(e, \mu)$.

- Binned maximum likelihood fit to the number of $b$-tagged jets.
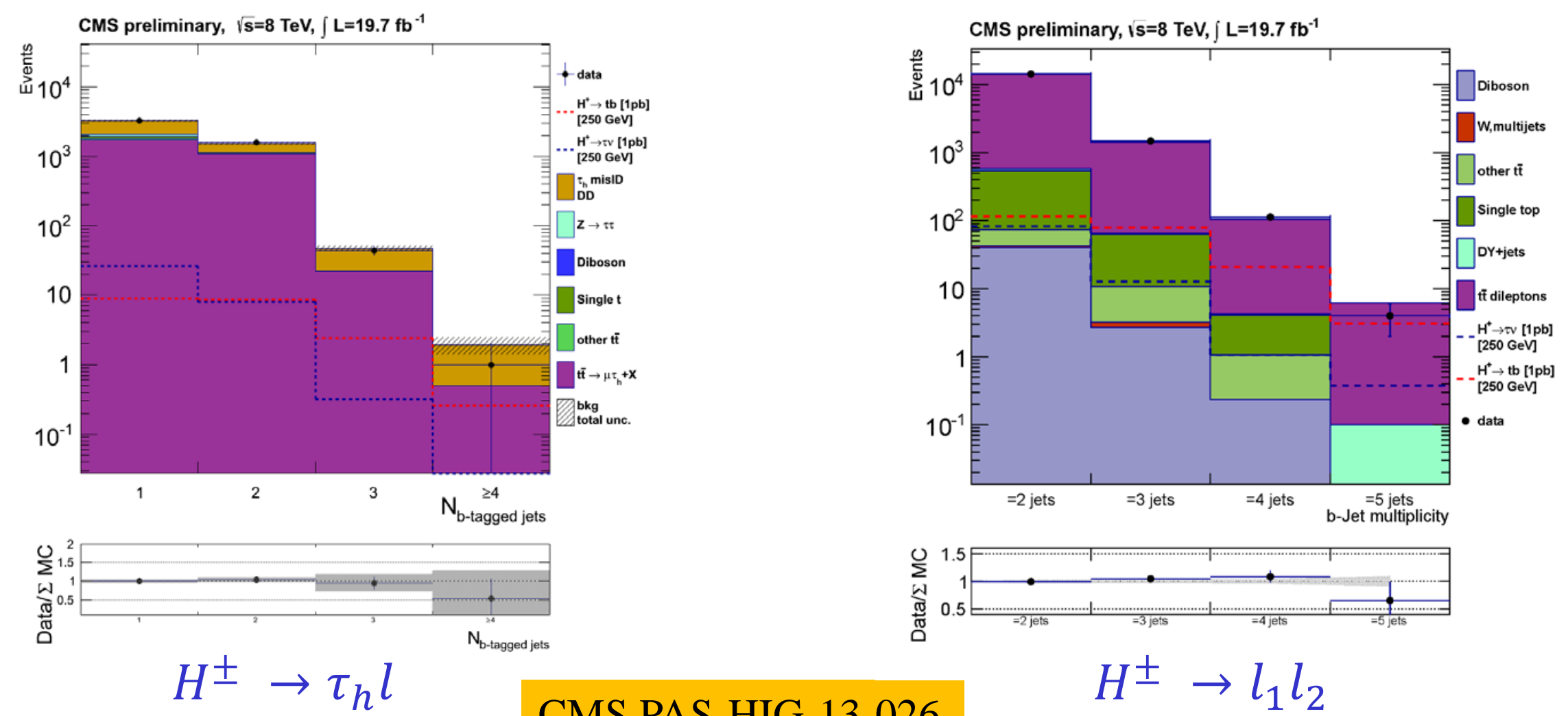

CMS PAS-HIG-13-026

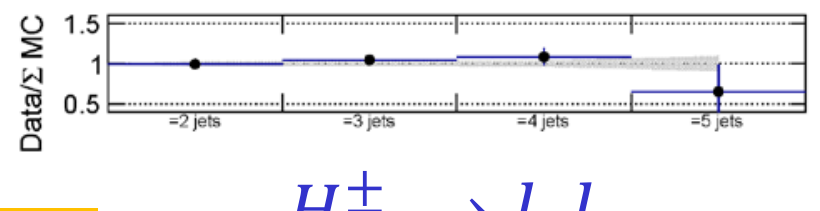

$$
H^{ \pm} \rightarrow l_{1} l_{2}
$$




\section{$H^{+} \rightarrow \tau^{+} v_{\tau}$ or $t \bar{b} ; m_{H^{+}}>m_{t}$ (CMS) [contd.]}
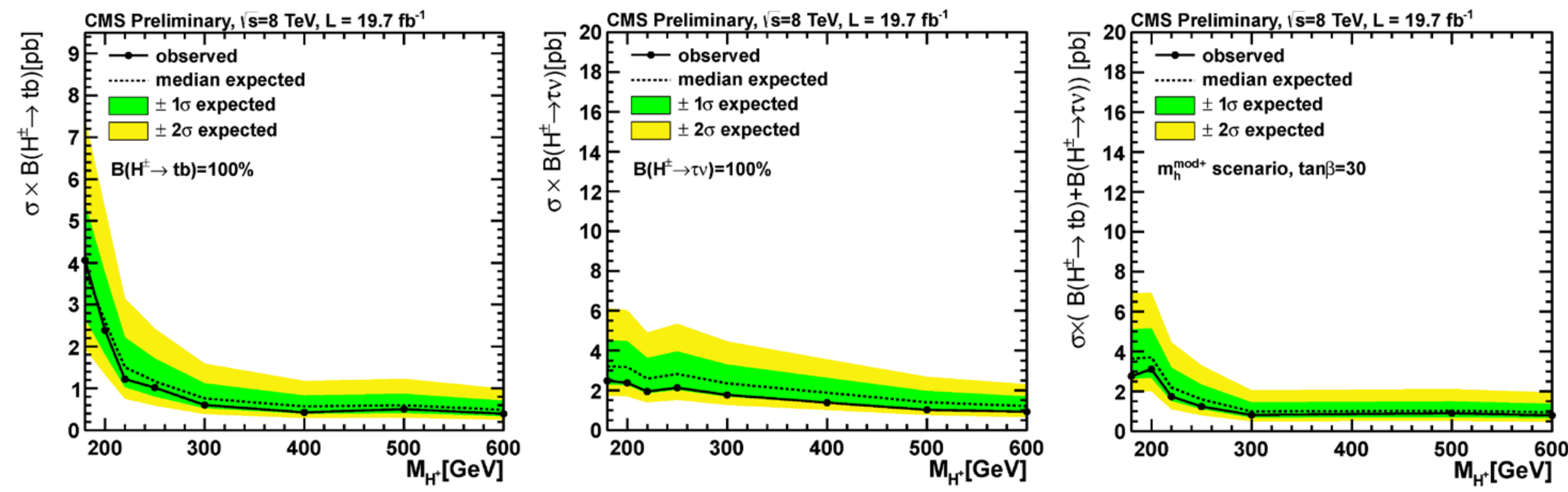

$$
\begin{gathered}
\sigma\left(p p \rightarrow H^{ \pm}\right) \times B\left(H^{+} \rightarrow t \bar{b}\right)<\sim 1-2 \mathrm{pb} \\
\sigma\left(p p \rightarrow H^{ \pm}\right) \times B\left(H^{+} \rightarrow \tau^{+} v_{\tau}\right)<\sim 4-5 \mathrm{pb} \\
\text { at } 95 \% \mathrm{CL} \text { for } 180 \mathrm{GeV}<m_{H^{+}}<600 \mathrm{GeV}
\end{gathered}
$$

CMS PAS-HIG-13-026 


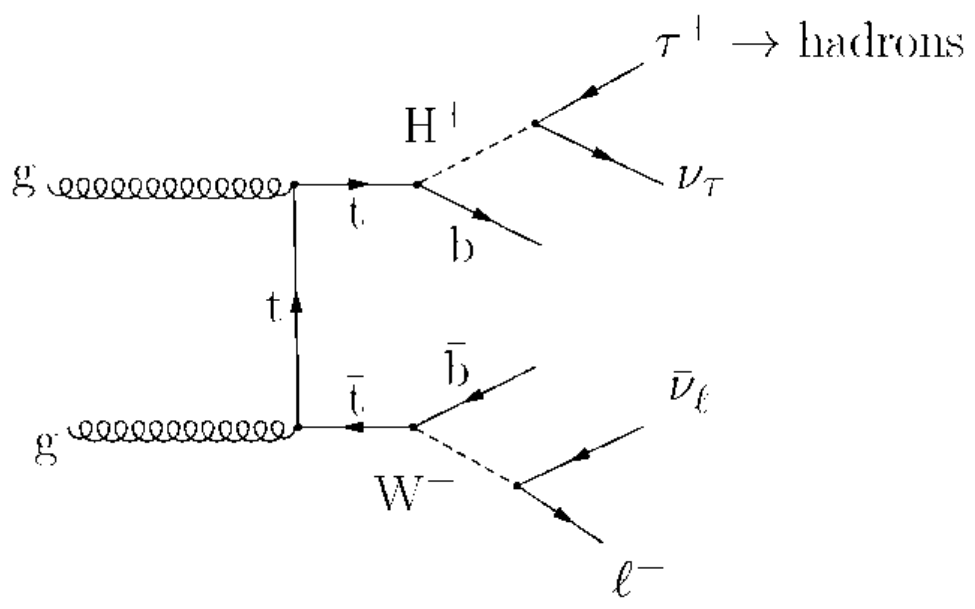

- Search in the $\tau_{h} l$ final state $l=e, \mu$

- Binned maximum likelihood fit to $R=p^{\text {lead.track }} / E_{\tau}$ distribution. This quantity is sensitive to $\tau$ polarization, which is different between $\tau^{\prime}$ 's from $H^{+}$and $W^{+}$decays.

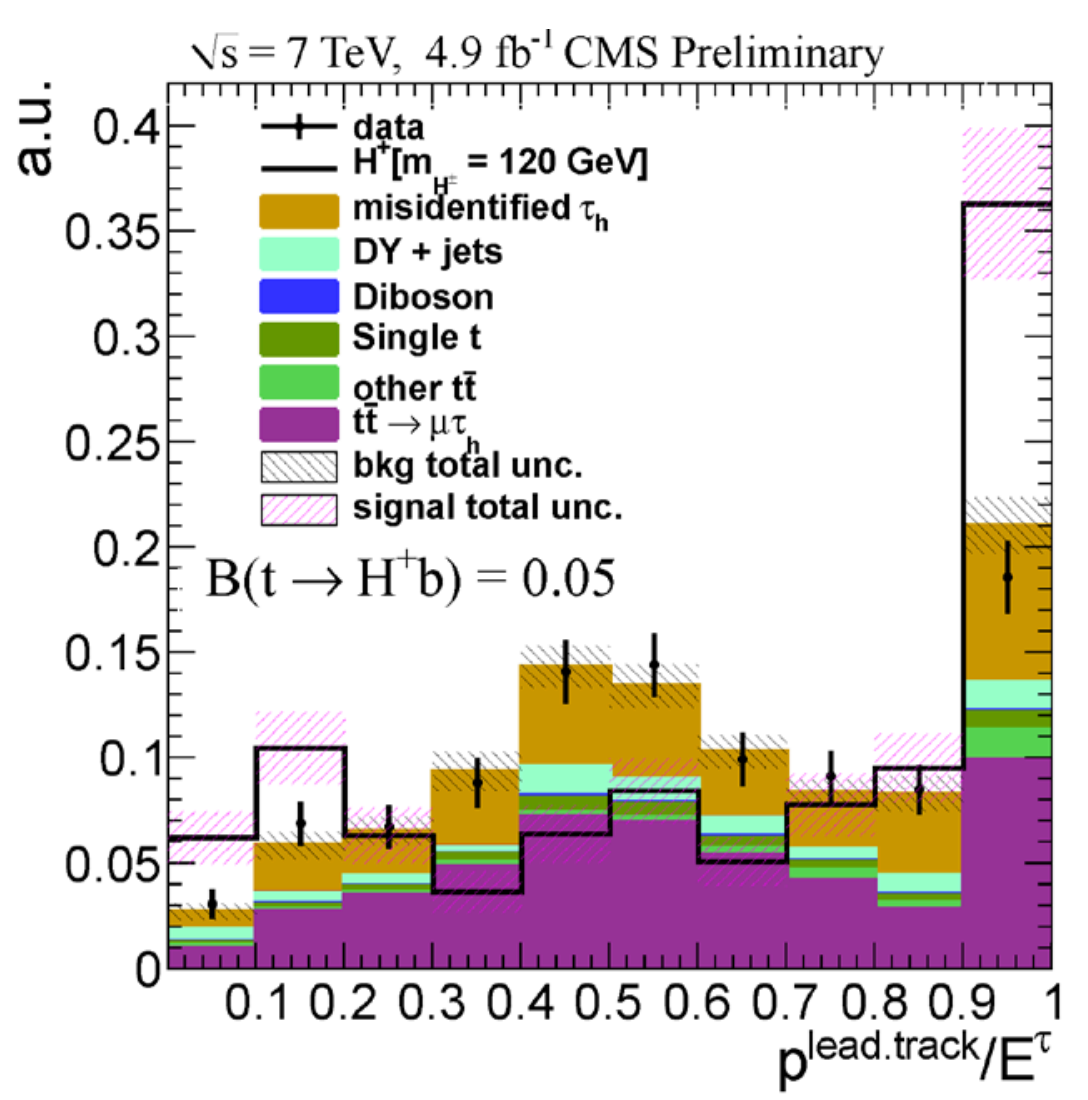




\section{$H^{+} \rightarrow \tau^{+} v_{\tau} ; m_{H^{+}}<m_{t}$ (CMS) [contd.]}

\begin{tabular}{|c|c|}
\hline Source & $\mathrm{N}_{\text {events }}$ ( \pm stat. \pm syst. $)$ \\
\hline \hline $\mathrm{HH}+\mathrm{HW}, m_{H^{+}}=120 \mathrm{GeV}, \mathcal{B}\left(\mathrm{t} \rightarrow \mathrm{H}^{+} \mathrm{b}\right)=0.05$ & $179.3 \pm 8.7 \pm 22.1$ \\
\hline \hline$\tau$ fakes (from data) & $222.0 \pm 11.4$ \\
$t \bar{t} \rightarrow W b W b \rightarrow(\mu v b)\left(\tau_{h} v b\right)$ & $304.7 \pm 2.8 \pm 25.9$ \\
$t \bar{t} \rightarrow W b W b \rightarrow(\ell v b)(\ell v b)$ & $21.4 \pm 0.7 \pm 6.9$ \\
$\mathrm{Z} / \gamma^{*} \rightarrow e e, \mu \mu$ & $0.4 \pm 0.4 \pm 0.1$ \\
$\mathrm{Z} / \gamma^{*} \rightarrow \tau \tau$ & $50.6 \pm 17.6 \pm 20.7$ \\
Single top & $26.6 \pm 1.2 \pm 3.3$ \\
VV & $4.4 \pm 0.5 \pm 0.7$ \\
\hline Total expected from SM & $630.1 \pm 17.9 \pm 46.9$ \\
\hline Data & 620 \\
\hline
\end{tabular}
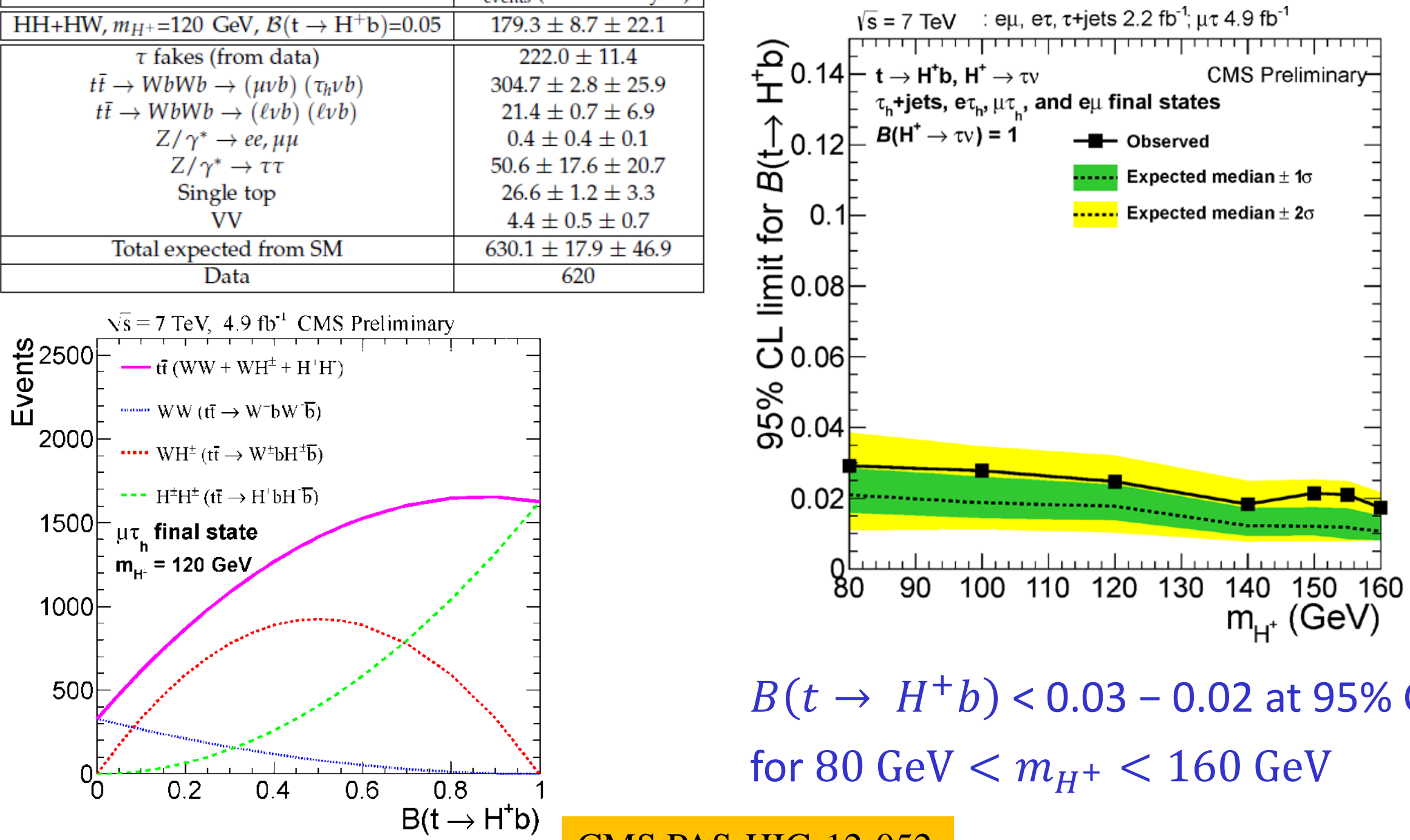

$B\left(t \rightarrow H^{+} b\right)<0.03-0.02$ at $95 \% \mathrm{CL}$ for $80 \mathrm{GeV}<m_{H^{+}}<160 \mathrm{GeV}$

CMS PAS-HIG-12-052 


\section{$H^{+} \rightarrow c \bar{s} ; m_{H^{+}}<m_{t}(\mathrm{CMS})$}
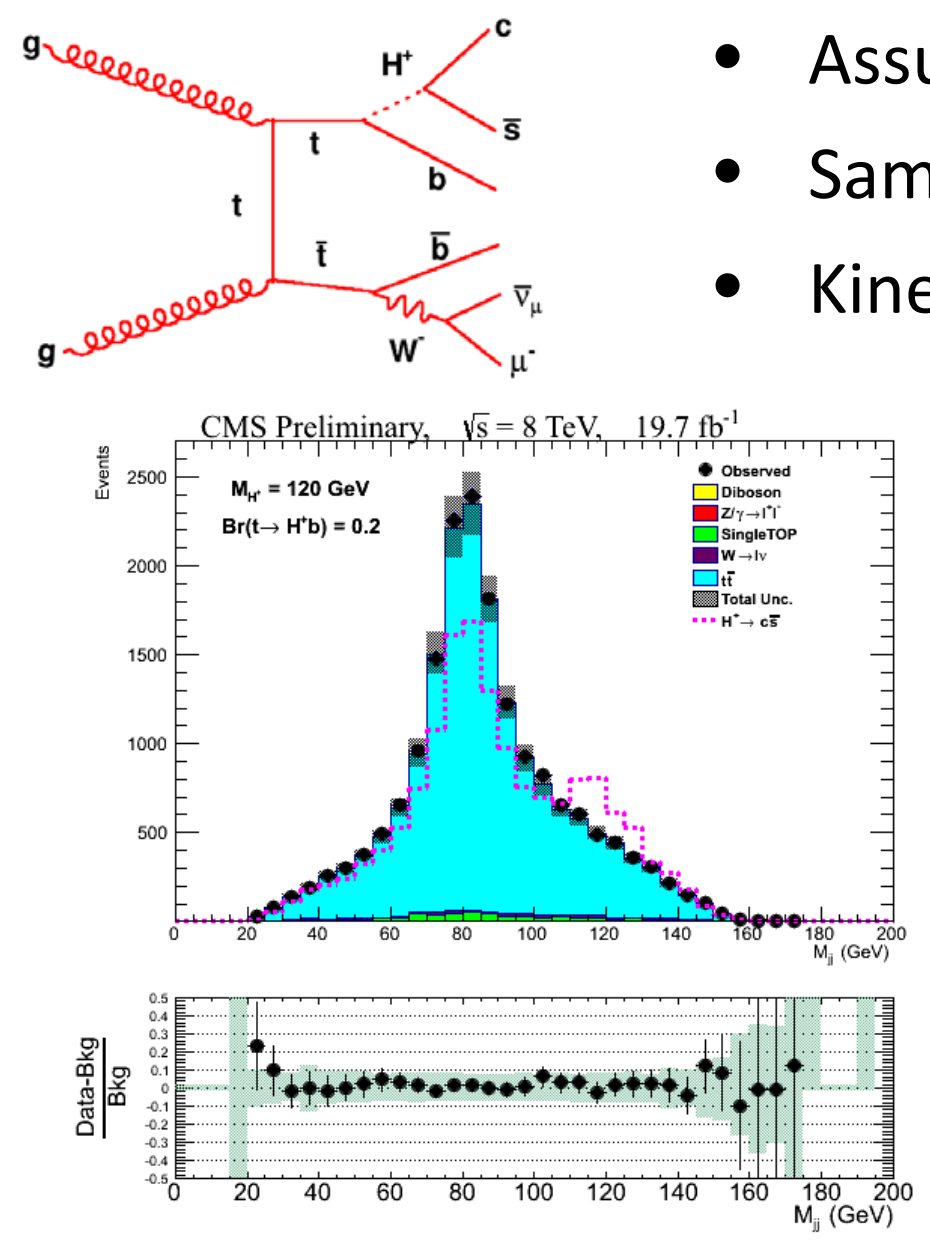

$B\left(t \rightarrow H^{+} b\right)<0.017-0.07$ at $95 \%$
- Assume $B\left(H^{+} \rightarrow c \bar{s}\right)=1$.

- Same final state as semileptonic decay of $t \bar{t}$ in SM

- Kinematic fit to both top candidates

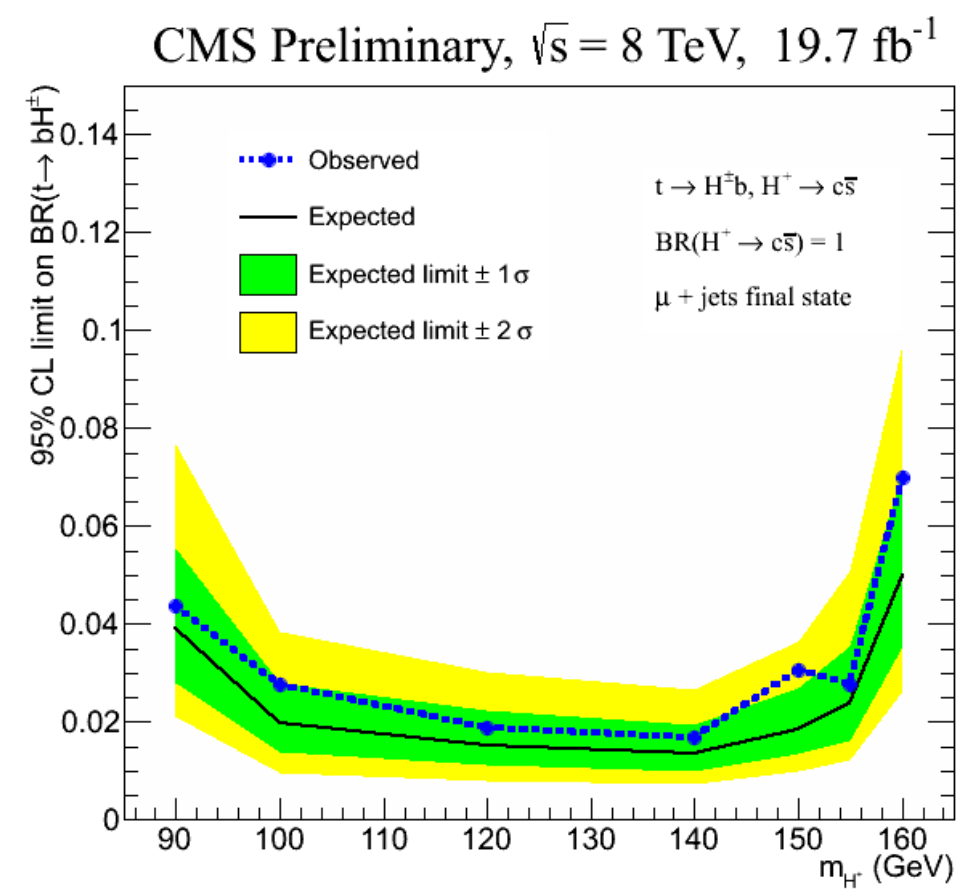

CMS PAS-HIG-13-035 


\section{lepton universality; $m_{H^{+}}<m_{t}$ (ATLAS)}

- Assume $B\left(H^{+} \rightarrow \tau^{+} v_{\tau}\right)=1$

- Compare $e+\tau_{h}, \mu+\tau_{h}$ yields to that of $e+\mu$

$$
R_{e+\mu}=\frac{\mathcal{N}\left(e+\tau_{\text {had }}\right)+\mathcal{N}\left(\mu+\tau_{\text {had }}\right)}{\mathcal{N}(e+\mu)+\mathcal{N}_{\mathrm{OR}}(\mu+e)}
$$

+

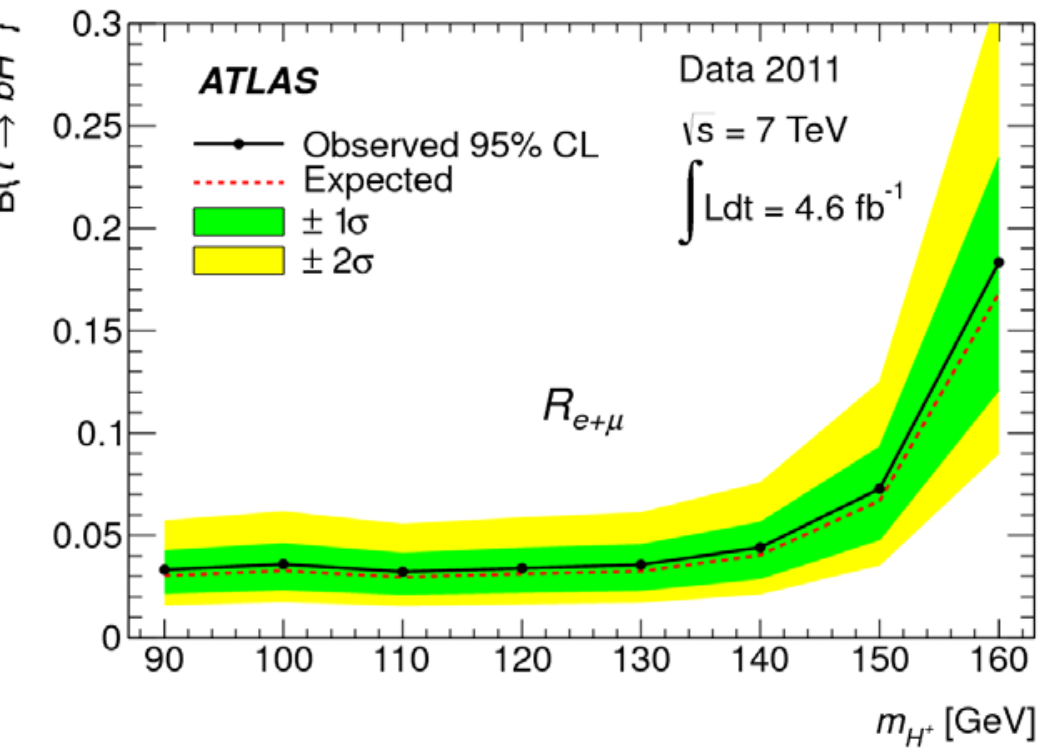

$B\left(t \rightarrow H^{+} b\right)<0.033-0.044$ at $95 \% \mathrm{CL}$

for $90 \mathrm{GeV}<m_{H^{+}}<140 \mathrm{GeV}$

JHEP03 (2013) 076
- Combined with $\tau_{h}+$ jets, gave best results for $\sqrt{s}=7 \mathrm{TeV}$.

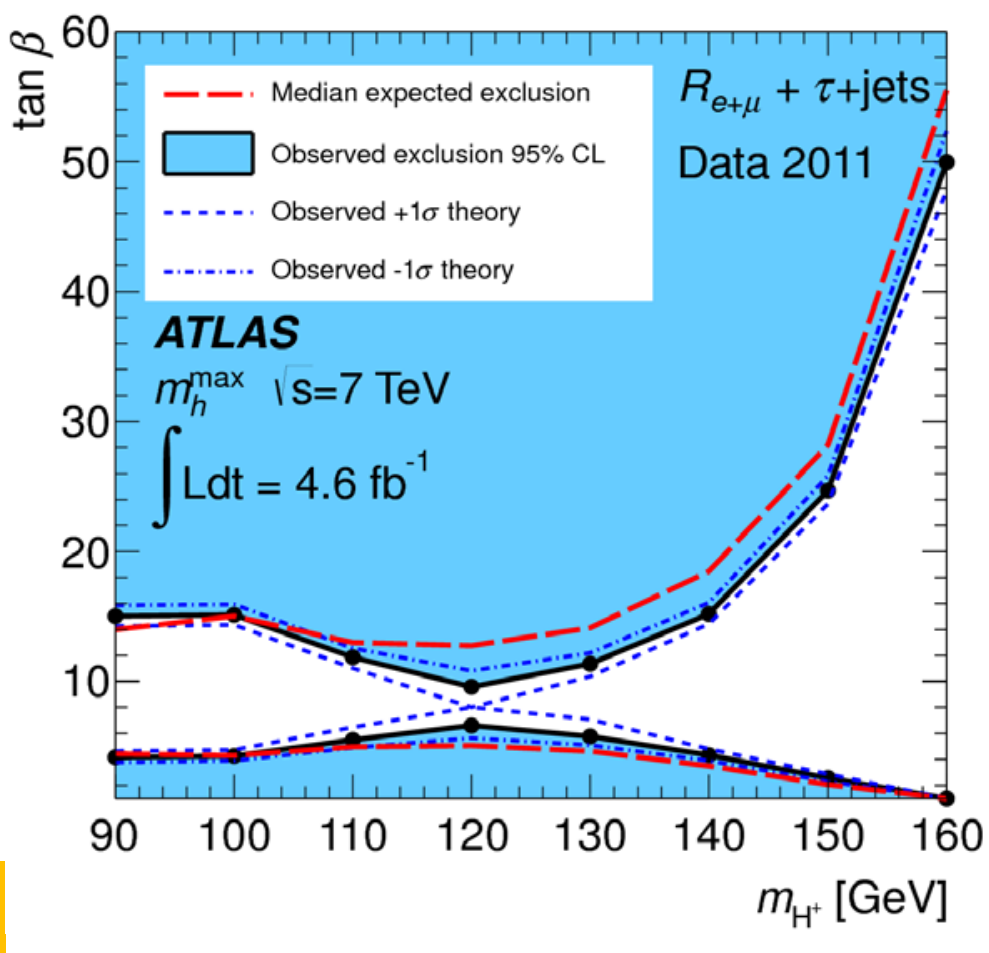




\section{$H^{0} \rightarrow H^{ \pm} W^{\mp} \rightarrow h^{0} W^{+} W^{-}$(ATLAS)}

- Look for

$$
H^{0} \rightarrow H^{ \pm} W^{\bar{\mp}} \rightarrow h^{0} W^{ \pm} W^{\mp} \rightarrow b \bar{b} l v j j
$$

- Fairly model-independent ( $A$ too heavy to appear in decay chain).

- Exploit $m_{b b}=m_{h^{0}}=125 \mathrm{GeV}$.

- Same final state as semileptonic decay of $t \bar{t}$ in SM.

- BDT trained at 36 mass points of $H^{0}$ to discriminate against $S M t \bar{t}$ events.

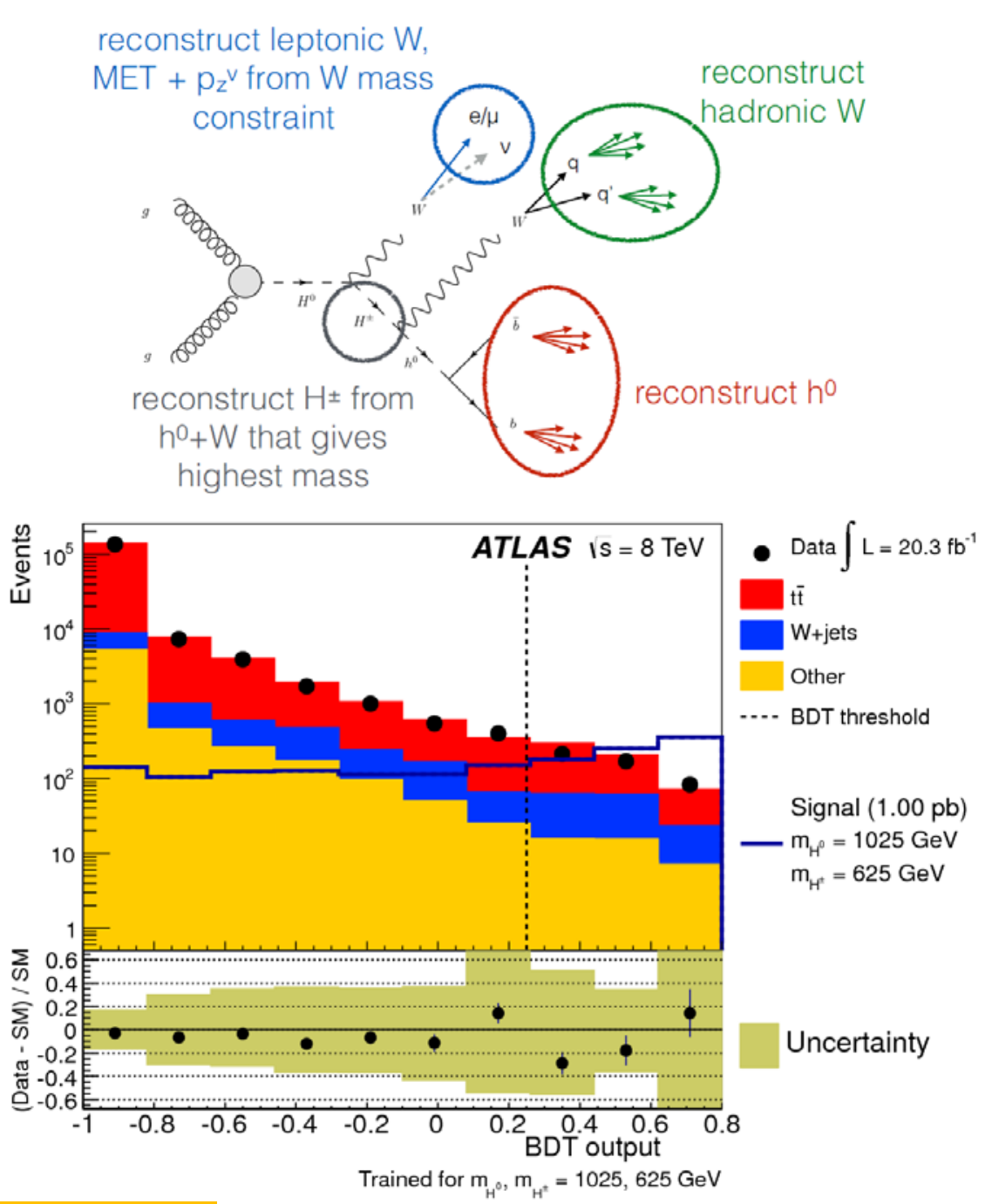

PRD 89 (2014) 032002 


\section{$H^{0} \rightarrow H^{ \pm} W^{\mp} \rightarrow h^{0} W^{+} W^{-}$(ATLAS) [contd.]}

The ratio of the observed 95\% CL upper limits on the cross section to the theoretical cross section for $g g \rightarrow H^{0} \rightarrow H^{ \pm} W^{\mp} \rightarrow$ $h^{0} W^{ \pm} W^{\mp} \rightarrow W^{ \pm} W^{\mp} b \bar{b}$ on the $\left[m_{H^{0}}, m_{H^{+}}\right]$plane.

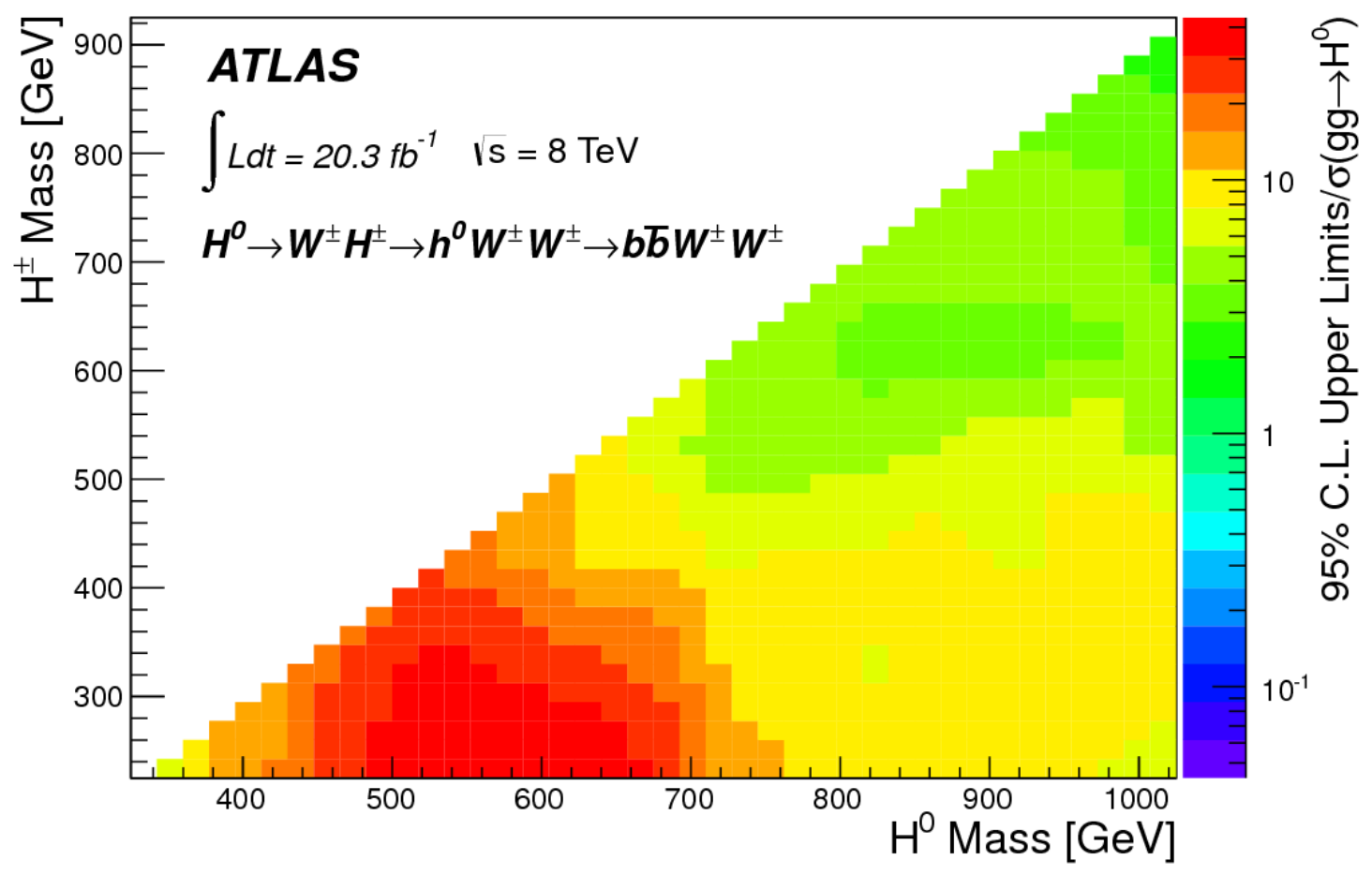

Observed upper limits are higher than theoretical expectation, but approaching it for larger $H^{0}$ masses.

PRD 89 (2014) 032002 


\section{Summary and outlook}

- Both ATLAS and CMS experiments have searched for production of charged Higgs bosons in $p p$ collisions up to $20(5) \mathrm{fb}^{-1}$ of data at $\sqrt{s}=8(7) \mathrm{TeV}$.

- Mostly via $H^{+} t b$ vertices so far, but investigations into alternatives have started.

- In the absence of evidence of signal, limits have been set on cross sections and/or branching fractions, interpreted to exclusion in $\left(m_{H^{+}}, \tan \beta\right)$ space.

- All previous direct results have been drastically improved.

- More (improved) results are expected soon.

- Close collaboration with theorists/phenomenologists to improve modeling.

- Higher production cross section and larger volume of data are expected to further improve results in Run 2. 


\section{Older result: $\boldsymbol{H}^{+} \rightarrow \boldsymbol{\tau}^{+} \boldsymbol{v}_{\tau}$ (ATLAS)}

\section{Interpretation in MSSM $m_{h}{ }^{\max }$ scenario}

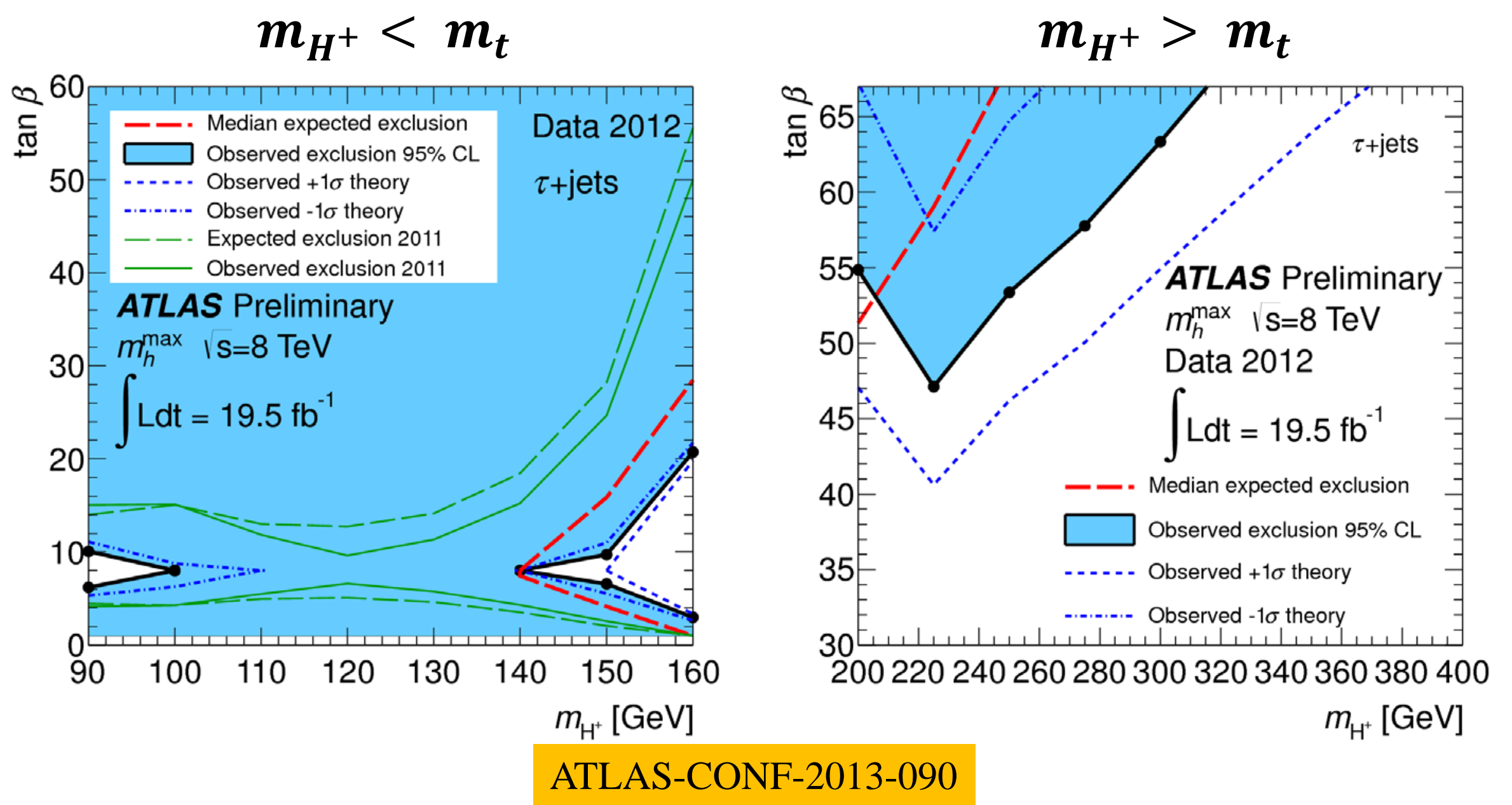

